\title{
A DOUBLE BLIND, CROSS-OVER TRIAL OF VALIUM IN THE TREATMENT OF SPASTICITY
}

\author{
By Margaret Corbett, M.B., B.S., H. L. Frankel, M.B., M.R.C.P., \\ and L. Michaelis, M.D. \\ National Spinal Injuries Centre, Stoke Mandeville Hospital
}

WHILE there is a good deal of international agreement that Valium has a quietening effect on spinal cord spasticity (Neill, I964; Cibeira et al., I964; Kerr, I966; Wilson \& McKechnie, I966; Cook \& Nathan, I967; Couvee et al., I968; Wilson, I970; Nathan, I970), it is still not clear how much of this effect is due to (I) suggestion, (2) sedation, (3) action on the spinal cord.

In this trial we have attempted to test (I) by Placebo, (2) by comparison with a known sedative, Amytal, and (3) by exclusion or separation of (I) and (2).

\section{MATERIAL AND METHODS}

A homogeneous group of patients was tested. All had traumatic lesions of the spinal cord and were four months or more after injury. They were divided into two sub-groups, physiologically complete and incomplete lesions. All had degrees of spasticity varying from the inconvenient to the disabling. All patients were volunteers who had been informed of the purpose of the trial. In order to minimise observer error or bias, assessment of the effect of treatment was made by six observers who each kept a protocol. This was later correlated and submitted for statistical analysis. Sheets for the protocol were distributed to the senior doctor, the patient, a senior physiotherapist-who did not treat the patient-a physiotherapist who did, and the junior doctor and ward sister in charge of the patient. All made daily entries on their respective sheets ( - worse, o no effect, + better, and ++ much better) except the senior doctor and senior physiotherapist who examined the patient once or twice a week. In spite of repeated attempts at obtaining daily entries, lapses of co-operation and changes of staff through illness or holidays left a number of gaps in the evidence collected. They were, however, not sufficiently serious to render the trial useless.

For each patient the trial took altogether six weeks, subdivided into three periods of a fortnight each. After three days without any drugs, each patient was given one tablet on the first day, one b.d. on the second day, one t.d.s. on each of the next three days. For the following three days he was given two tablets t.d.s. and for the last three days, three tablets t.d.s.

The tablets for each fortnightly period were taken from three identical bottles containing tablets of identical appearance. Placebo, Amytal $30 \mathrm{mg}$., and Valium $5 \mathrm{mg}$. were randomly distributed into bottles I, II and III, none of the participants knowing which contained which. The key was held by the manufacturer's laboratory. A copy was kept in a sealed envelope in the department in case of emergency.

The reason for this gradual increase in dosage was that experience had shown very varied responses to Valium in different patients, drowsiness in particular being 
caused in some after one dose of $2 \mathrm{mg}$, while others could take $45 \mathrm{mg}$. without side-effect.

Even in a large department it was not easy to find the required number of patients who could be observed for six weeks. In all, 22 patients could be included in the trial (20 male, 2 women) of whom 15 were tetraplegic and 7 paraplegic. Fourteen had complete lesions and 8 had incomplete lesions.

\section{STATISTICAL METHODS}

The available scores for each treatment were standardised and weighted and presented as:

(I) Means of total weighted scores (standardised).

(2) Mean ranks.

(3) The results of Friedman significance tests (Siegel, I956; Miller, I966).

\section{RESULTS}

Results of mean total weighted scores are given in Table I.

Figure I shows the mean ranks.

Results of Friedman significance test are given in Table II. This shows that the senior doctor found a significant difference between Valium and either Placebo

TABLE I

Means of Total Weighted Scores (Standardised)

\begin{tabular}{|l|c|c|c|c|}
\hline \multicolumn{1}{|c|}{ Assessment } & Valium & Amytal & Placebo & No. of patients \\
\hline Senior doctor & $25 \cdot 9 \mathrm{I}$ & $2 \mathrm{I} \cdot 08$ & $2 \mathrm{I} \cdot 6 \mathrm{I}$ & I9 \\
Senior physiotherapist & $23 \cdot 79$ & $2 \mathrm{I} \cdot 86$ & $\mathrm{I} 7.92$ & I I \\
Doctor/sister & 27.47 & I9.55 & 24.52 & I9 \\
Patient & $25.7 \mathrm{I}$ & 23.04 & I9.89 & I I \\
Physiotherapist & $2 \mathrm{I} \cdot 68$ & I9.I8 & I8.49 & 9 \\
\hline
\end{tabular}

TABLE II

Results of Significance Test-Friedman

\begin{tabular}{|l|c|c|l|}
\hline \multicolumn{1}{|c|}{ Assessment } & $\chi^{2}$ & \multicolumn{1}{|c|}{$\mathrm{P}$} & \multicolumn{1}{|c|}{ Significant difference between } \\
\hline Senior doctor & $7.92 \mathrm{I}$ & $>0.02$ & Valium and either Amytal or Placebo \\
Senior physiotherapist & $7.09 \mathrm{I}$ & $>0.05$ & Valium and Placebo \\
Doctor/sister & 6.737 & $>0.05$ & Valium and Amytal \\
Patient & 2.364 & N.S. & None \\
Physiotherapist & $\mathrm{I} \cdot 055$ & N.S. & None \\
\hline
\end{tabular}

or Amytal (Valium superior). The senior physiotherapist found a significance between Valium and Placebo (Valium superior) and the combined junior doctor/ ward sister assessment showed a significance between Valium and Amytal (Valium 
superior). An attempt was made to see whether patients with complete cord lesions reacted differently from those with incomplete lesions, but the numbers were small and no conclusions could be drawn.

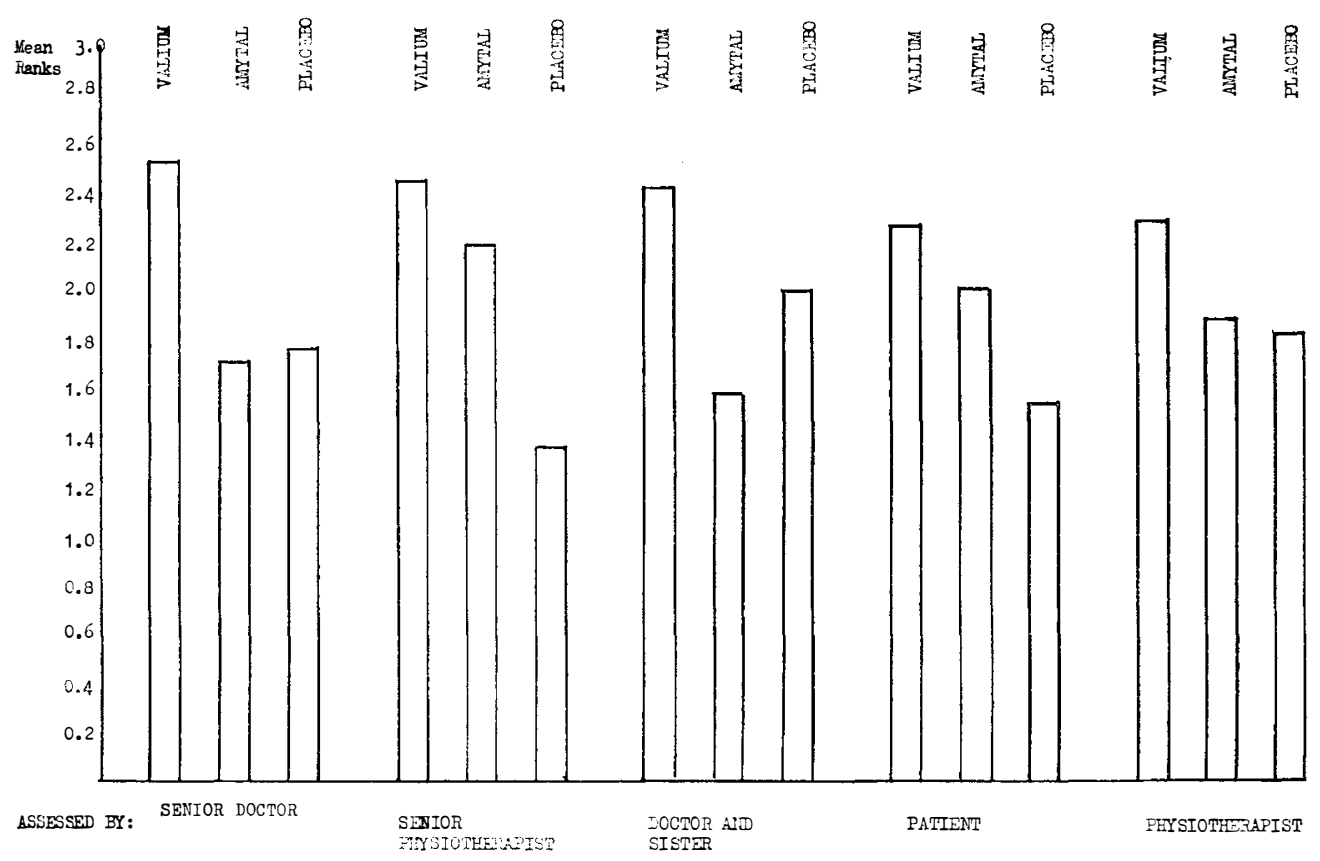

\section{SIDE-EFFECTS}

Drowsiness was reported during II treatments in 7 patients. Six reports were when the patient was on Valium, two reports were while the patients were on Amytal and three reports were while on Placebo. Four patients reported drowsiness while on two different treatments, the other three reported it during only one treatment. In only one patient did the dose have to be reduced from three tablets t.d.s. - that patient was taking Valium at the time and could not tolerate $45 \mathrm{mg}$. daily but could tolerate $30 \mathrm{mg}$. daily. All other patients tolerated three tablets t.d.s. of all three treatments.

In addition, one patient complained of drowsiness between treatments while he was taking no tablets.

No other side-effects were noted.

\section{DISCUSSION}

Previous double blind trials have demonstrated the superiority of Valium over Placebo in spinal spasticity (Neill, 1964; Kerr, 1966; Wilson \& McKechnie, I966). However, we suspected that many patients might recognise the Valium due to its sedative effect and for this reason included a known sedative, Amytal, in the trial. Our results indicated that in the doses used Valium is superior to Amytal and Placebo in reducing spasticity resulting from spinal cord lesions. 
Although Valium is shown to be superior to Amytal and Placebo this does not imply that Valium is an altogether satisfactory treatment for spasticity. In only two patients did Valium give the hoped-for relief of spasticity. In all other cases showing improvement the relief was partial. There is still a need for all other methods of treatment including physiotherapy and hydrotherapy.

It was expected that most of the patients would become drowsy on the full doses of Valium and Amytal and it was our common clinical expectation that nearly all patients would be very drowsy on $45 \mathrm{mg}$. per day of Valium. This did not occur; in only six patients did this dose of Valium give rise to drowsiness and in only one patient did we have to reduce the dose. The low incidence of sideeffects in this double blind trial indicates that the side-effects as well as the therapeutic effects of a drug may be influenced by suggestion.

This method of testing a drug has been difficult and time-consuming. The length of time-six weeks for each patient-was due firstly to our ascending dosage system and secondly due to the double cross-over nature of the trial. For future trials of new drugs we intend to have only four independent observers and use a double blind trial of the new drug against Valium which we currently consider to be the 'standard drug'.

\section{SUMMARY}

A double blind cross-over trial of Valium against Amytal and Placebo was carried out on 22 patients with spasticity due to spinal cord injuries. Observations were made by six independent observers. Valium was significantly more effective than Amytal or Placebo. There was a low incidence of side-effects.

Acknowledgements. We should like to thank all patients and staff for their willing co-operation, and Messrs. Roche for the preparation of the trial tablets and financial assistance.

\section{REFERENCES}

Cibeira, J. B., Buzzi, A. \& Dominelli, J. C. (1964). Pren. Med. Argent. 5I, 1544.

Cook, J. B. \& Nathan, P. W. (1967). F. Neurol. Sci. 5, 33.

Couvee, L. M. J., VAn Der LaARse, W. D. \& Oosterveld, W. J. (1968). Int. F. Paraplegia, 6, 96.

KerR, W. G. (1966). Int. F. Paraplegia, 4, I49.

MilleR, R. J. (I966). Simultaneous Statistical Inference. McGraw-Hill.

Nathan, P. W. (1970). F. Neurol. Sci. 10, 33.

NeILl, R. W. K. (I964). Annals of Physical Med., Supplement, p. 33.

Siegel, S. (1956). Non Parametric Statistics for the Behavioural Sciences. McGraw-Hill.

Wilson, L. A. (1970). Gerontologia Clinica, 12, I68.

Wilson, L. A. \& McKechnie, A. A. (I966). Scot. Med. F. II, 46. 